\title{
Action, State and Effect Metrics for Robot Imitation
}

\author{
Aris Alissandrakis, Chrystopher L. Nehaniv and Kerstin Dautenhahn
}

\begin{abstract}
This paper addresses the problem of body mapping in robotic imitation where the demonstrator and imitator may not share the same embodiment (degrees of freedom (DOFs), body morphology, constraints, affordances and so on). Body mappings are formalized using a unified (linear) approach via correspondence matrices, which allow one to capture partial, mirror symmetric, one-to-one, one-to-many, many-to-one and many-to-many associations between various DOFs across dissimilar embodiments. We show how metrics for matching state and action aspects of behaviour can be mathematically determined by such correspondence mappings, which may serve to guide a robotic imitator. The approach is illustrated in a number of examples, using agents described by simple kinematic models and different types of correspondence mappings. Also, focusing on aspects of displacement and orientation of manipulated objects, a selection of metrics are presented, towards a characterization of the space of effect metrics.
\end{abstract}

\section{INTRODUCTION}

$\mathbf{I}$ MITATION is a powerful learning tool when a number of agents interact in a social context. The demonstrator and imitator agents may or may not belong to the same species (a parent teaching a child, a human training an animal) or even be biological and artificial entities (e.g. in humanrobot interaction). The latter is a very interesting paradigm explored in computer science and robotics, with researchers influenced by work on biology, ethology and psychology in order to design controllers that would allow their robots to be programmed and learn more easily and efficiently [1], [2], [3], [4], [5], [6].

A fundamental problem when learning how to imitate is to create an appropriate (partial) mapping between the actions afforded by particular embodiments to achieve corresponding states and effects by the model and imitator agents (solving a correspondence problem) [7]. The solutions to the correspondence problem will depend on the subgoal granularity and the metrics used to evaluate the similarity between actions, states and/or effects, resulting in qualitative different imitative behaviours [8], [9]. The related problem of what to imitate addresses the choice of metrics and subgoal granularity that should be used for imitating, depending on the context. See [10], [11] for robotic examples and [12], [13], [14] for ethological and psychological aspects.

In the current work, we present a novel generic approach to the correspondence problem, via body-mapping for the

The work described in this paper was conducted within the EU Integrated Project COGNIRON ("The Cognitive Robot Companion") and supported by the European Commission Division FP6-IST Future and Emerging Technologies under Contract FP6-002020.

The authors are with the School of Computer Science, Adaptive Systems Research Group, University of Hertfordshire, College Lane, Hatfield, Hertfordshire AL10 9AB, U.K. a.alissandrakis@herts.ac.uk cases of state and/or action matching. In particular, partial, relative and mirror matching all arise as special cases of such correspondence mappings. Moreover, an infinite set of metrics (parameterized by correspondence matrices) for imitation performance are induced via such body correspondences. ${ }^{1}$ This contributes towards a characterization of types of matching in social learning, extending [8], [15], [16]

Towards a characterization of the space of effect metrics, a selection of metrics is also presented, that focuses on aspects of displacement and orientation of manipulated objects.

\section{Correspondence Problem}

For similar embodiments, addressing the correspondence problem seems to be straightforward (although it actually involves deep issues of perception and motor control). But once the assumption that the agents belong to the same 'species', i.e. have sufficiently similar bodies and an equivalent set of actions, is dropped, as with a robot imitating a human, the problem becomes more difficult and complex. Even among biological agents, individual differences in issues of perception, anatomy, neurophysiology, and ontogeny can create effectively dissimilar embodiments between members of the same species. A close inspection of seemingly similar artificial agent embodiments can yield similar conclusions due to issues like individual sensor and actuator differences (hardware) or the particular representations and processing that these agents employ (software). In our setting, it will be desirable to have different kinds of agents in the learning process, i.e. humans and robots interacting socially.

The following statement of the correspondence problem [17], [18], [19] draws attention to the fact that the model and imitator agents may not necessary share the same morphology or may not have the same affordances:

Given an observed behaviour of the model, which from a given starting state leads the model through a sequence (or hierarchy [or program]) of $s u b$ goals in states, action and/or effects, one must find and execute a sequence of actions using one's own (possibly dissimilar) embodiment, which from a corresponding starting state, leads through corresponding sub-goals - in corresponding states, actions, and/or effects, while possibly responding to corresponding events.

In this approach, an imitator can map observed actions of the model agent to its own repertoire of actions using the correspondence found by solving the correspondence problem, as

${ }^{1}$ That is, to say that a correspondence mapping "induces" a metric means exactly that it mathematically determines the metric. 
constrained by its own embodiment and by context [17], [18], [19]. Qualitatively different kinds of social learning result from matching different combinations of matching actions, states and/or effects at different levels of granularity [15].

Artificial agents that have the ability to imitate may use (perhaps more than one) metric to compare the imitator agent's own actions, states and effects with the model's actions, states and effects, in order to evaluate the imitation attempts and discover corresponding actions that they can perform to achieve a similar behaviour. The choice of metrics used is therefore very important as it will have an impact on the quality and character of the imitation. Many interesting and important aspects of the model behaviour need to be considered, as the metrics capture the notion of the salient differences between performed and desired actions and also the difference between attained and desired states and effects [18], [19]. The choice of metric determines, in part, what will be imitated, whereas solving the correspondence problem concerns how to imitate [20]. In general, aspects of action, state and effect as well as the level of granularity (what to imitate) do all play roles in the choice of metric for solving the problem of how to imitate [18], [9], [10]. On-going research is thus addressing the complementary problem of how to extract sub-goals and derive suitable metrics automatically from observation [18], [15], [10], [21].

\section{DIFFERENT BODIES}

Different agent bodies can be described as simplified kinematic models, comprising of a rooted acyclic connected graph of segments. Each segment has a base and a tip end, and can described by of the quintuple $\left(i, \ell_{i}, p_{i}, \theta_{i}, \phi_{i}\right)$, where

- $i$ is the index number of the segment,

- $\ell_{i}$ is segment length,

- $p_{i}$ is the index number of the parent segment,

- and $\theta_{i}$ and $\phi_{i}$ are the azimuth and polar angles for the spherical coordinates $\left(l_{i}, \theta_{i}, \phi_{i}\right)$ that indicate how the segment is positioned in $3 \mathrm{D}$ space (relative to the end of its parent segment). ${ }^{2} \mathrm{NB}$ : In general the range of the angles $\theta_{i}$ and $\phi_{i}$ may be constrained within given respective ranges.

The values of $\theta_{i}$ and $\phi_{i}$ are relative for each segment, but absolute angles for segment $i, \Theta_{i}$ and $\Phi_{i}$, can be obtained inductively starting from the next segment after the root segment. $^{3}$

The state (or posture) of such a kinematic model can be defined as the vector $S$ containing the values of the degrees of freedom (DOF), i.e. here the values of the azimuth and polar angles for each segment. For the rest of the paper, the notation $S_{j}$ will be used to refer to the state value of the $j^{t h}$ DOF of an agent.

\footnotetext{
${ }^{2}$ Please note that the choice of using spherical coordinates (with two angles to describe the position of each segment) is done here for simplicity. In general, the three Euler angles could also be used.

${ }^{3}$ For mathematical convenience, the root node is treated as a segment of length $\ell_{0}=0$, but $\theta_{0}$ and $\phi_{0}$ can have non-zero values, to orient the entire model. For expository purposes, without loss of generality, in this article we ignore the latter possibilities $\left(\theta_{0}=0, \phi_{0}=0\right)$.
}

An action can be thought as a motion, or the amount of change in the DOFs required so that one posture transforms to another, and can be defined as the difference between two consecutive state vectors $S$ and $S^{\prime}: A=S^{\prime}-S$. For the rest of the paper, the notation $A_{j}$ will be used to refer to the value by which the $j^{\text {th }}$ DOF of an agent must change, for the state value $S_{j}$ to become $S_{j}^{\prime}$.

For effects, see section $\mathrm{V}$ below.

\section{State And Action Metrics}

To evaluate the similarity of behaviour, with respect to states and actions, between an agent $\beta$ imitating another agent $\alpha$, we need to define and use appropriate metrics. For the moment let us assume that both agent embodiments have the same number of DOFs, $n$.

A first global state metric can be defined as

$$
\mu_{\text {state }}=\sum_{j=1}^{n}\left|S_{j}^{\alpha}-S_{j}^{\beta}\right| \text {, }
$$

where $S_{j}^{\alpha}$ and $S_{j}^{\beta}$ are the values of the state vectors for the two agents.

Similarly, a first global action metric can be defined as

$$
\mu_{\text {action }}=\sum_{j=1}^{n}\left|A_{j}^{\alpha}-A_{j}^{\beta}\right|,
$$

where $A_{j}^{\alpha}$ and $A_{j}^{\beta}$ are the values of the action vectors for the two agents.

An agent performing actions so as to minimize one (or a weighted combination) of these two metrics would successfully imitate a demonstrator in respect to states and/or actions. In the following subsections, some more complex action/state metrics are defined.

\section{A. Correspondence Mapping}

For two agents, demonstrator $\alpha$ and imitator $\beta$ with $n$ and $m$ DOFs respectively, a $n \times m$ correspondence matrix can be defined as

$$
\mathcal{C}=\left[\begin{array}{cccc}
w_{1,1} & w_{1,2} & \ldots & w_{1, m} \\
w_{2,1} & w_{2,2} & \ldots & w_{2, m} \\
\vdots & \vdots & \ddots & \vdots \\
w_{n, 1} & w_{n, 2} & \ldots & w_{n, m}
\end{array}\right]
$$

where the $w_{i, j}$ values are real-valued weights, determining how the $j^{\text {th }}$ DOF of the imitator $\beta$ depends on the $i^{\text {th }}$ DOF of the demonstrator $\alpha$. The $j^{\text {th }}$ column of the matrix can be thought as a vector indicating how the DOFs of the demonstrator influence the $j^{\text {th }}$ DOF of the imitator. Depending on how many of the weights have a non-zero value, this correspondence mapping can be one-to-one, oneto-many (or many-to-one) or many-to-many. If partial body imitation is desired, some DOF of the imitator (and/or the demonstrator) can be omitted by setting an entire column (resp. row) to zero in the correspondence matrix.

Assuming both agents share the same embodiment (and as a result have the same number of DOFs), a simple example of a one-to-one correspondence mapping would be using the 

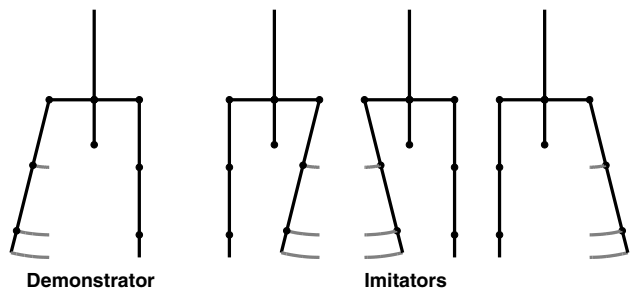

Fig. 1. Examples of symmetry via correspondence mapping. The figure shows a demonstrator (left) and three imitators, facing the reader, each with an upper human torso embodiment. The demonstrator is moving its right arm to its left. Each of the three imitators are using different correspondence mappings: mapping the demonstrator's right arm to the left arm of the imitator (second from the left), using a weight of minus one, but maintaining the same arm mapping (second from the right) and finally both mapping the demonstrator's right arm to the left arm of the imitator and using a weight of minus one (right). The grey lines trace the movement of the arms.

identity matrix as a correspondence matrix. Alternatively, if some mirror symmetry is wanted, then the DOFs for the right arm and leg of the demonstrator (see example in Fig. 1, left) could be mapped to the DOFs for the left arm and leg of the imitator, and vice versa (Fig. 1, second from the left). Another possible form of symmetry results from mapping some of the demonstrator's DOF using a weight of minus one (e.g. if the demonstrator raises its hand, the imitator should lower its hand or if the demonstrator turns its head to the left the imitator should turn to the right, see example in Fig. 1, second from right).

If the agents do not have the same number of DOFs (or depending on their particular morphology), it may be useful to map a single DOF to many DOFs. For example, consider correspondences between a human body as model to a dolphin-like imitator: ${ }^{4}$ a dolphin using its mouth corresponding to either human arm (grasping an object), or its tail to toss a ball back to a human that used both arms comprise real-world examples of many-to-one mappings. These two examples also illustrate that the correspondence need not to be static - the human hand(s) are mapped to different dolphin body parts in each case - but can be adapted depending on the context and the tasks involved.

\section{B. Induced State and Action Metrics}

The metric definitions (1) and (2) are appropriate for the most simple one-to-one mapping (the identity mapping), with both agents sharing the same number of DOFs (and probably a very similar morphology). But in general, using a correspondence matrix, other metric definitions can be induced.

By multiplying an appropriate correspondence matrix $\mathcal{C}$ with the state and action vectors $S^{\alpha}$ and $A^{\alpha}$ of the demonstrator respectively, two new vectors in imitator coordinates can be produced:

$$
\begin{aligned}
& \mathcal{S}=S^{\alpha} \times \mathcal{C} \\
& \mathcal{A}=A^{\alpha} \times \mathcal{C}
\end{aligned}
$$

${ }^{4}$ Different mappings do appear to be employed by real-life dolphins in imitating humans [22].
Combining (1) and (3) gives

$$
\mu_{\text {state }}^{\mathcal{C}}=\sum_{j=1}^{m}\left|\mathcal{S}_{j}-S_{j}^{\beta}\right| \epsilon_{j},
$$

where the corrective term

$$
\epsilon_{j}=\left\{\begin{array}{l}
0 \text { if } \sum_{\substack{i=1 \\
1 \text { otherwise }}}^{n} w_{i, j}^{2}=0
\end{array}\right.
$$

takes the value zero if the $j^{\text {th }}$ column of the correspondence matrix contains only zeros (effectively omitting the imitator's $j^{\text {th }}$ DOF). Intuitively, the components of $\mathcal{S}$ and $\mathcal{A}$ (for such $\left.\epsilon_{j} \neq 0\right)$ can be thought as the current subgoal state and action target values.

Finally, combining (2), (4) and (5) gives:

$$
\mu_{\text {action }}^{\mathcal{C}}=\sum_{j=1}^{m}\left|\mathcal{A}_{j}-A_{j}^{\beta}\right| \epsilon_{j}
$$

These new $\mu_{\text {state }}^{\mathcal{C}}$ and $\mu_{\text {action }}^{\mathcal{C}}$ metrics are called the induced state and action metrics for the linear correspondence C.

Depending on the correspondence mapping used, a plethora of new complex metrics (also allowing for dissimilar embodiments) can be induced considering state or action aspects. The next subsection will illustrate a variety of examples.

\section{Mapping Across Dissimilar Bodies}

For a given demonstrator and imitator embodiment pair, the imitator attempts to match the behaviour of the demonstrator by minimizing a given metric (or a combination of metrics). This can be done continuously (immediate imitation) or after the completion of the demonstration (deferred imitation) [23]. Moreover, the granularity or "fineness" of the matching of actions, states and/or effects determines a sequence of subgoals for the imitator to achieve, and the appropriate level of granularity may be different depending on context and task. Different correspondence mappings can be defined between the two agents, yielding qualitatively different types of matching behaviours.

Note that the various mappings presented here in the following sections are intended as illustrative examples, currently 'given' (i.e. hand-coded) and not derived by automated machine-learning techniques.

1) Identity and Mirror Symmetry Mappings: Two examples of imitation across similar embodiments are shown in Fig. 2. Both demonstrator and imitator are humanoid. In the first example the identity correspondence mapping is used. In the second example, using the same demonstration, symmetry is achieved by mapping the left body parts of the demonstrator to the right body parts of the imitator and vice versa (see also examples in Fig. 1). 

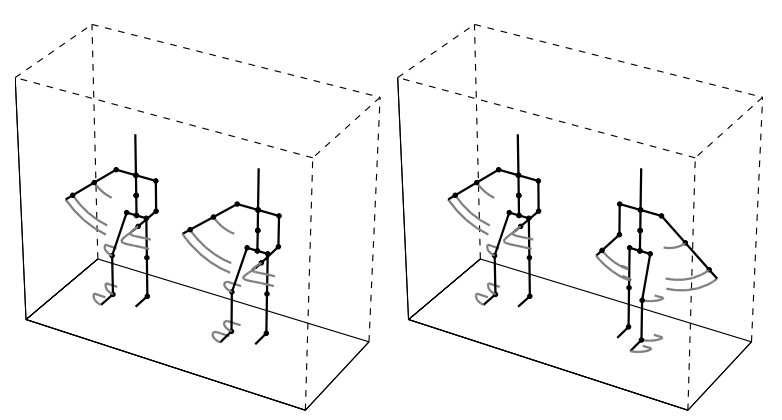

Fig. 2. Two examples of imitation across similar embodiments (humanoid). Both demonstrator (left in both examples) and imitator (right in both examples) share the same humanoid embodiment. In the example on the left, the identity mapping is used as the correspondence mapping. In the example on the right, the left arm and leg of the demonstrator are mapped on the right arm and leg of the imitator (and vice versa) with a weight of minus one, resulting in a mirror symmetry. The grey traces visualize the body part trajectories.

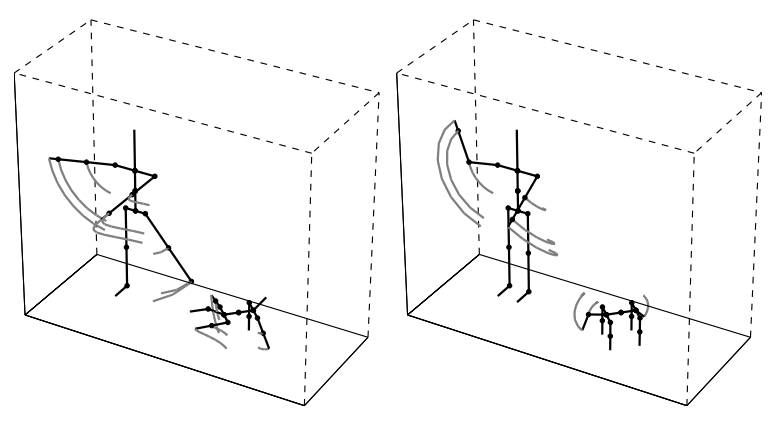

Fig. 3. Two examples of imitation across dissimilar embodiments (humanoid and dog-like). The demonstrator (left in both examples) is embodied as a humanoid, while the imitator (right in both examples) is embodied as a dog-like AIBO robot.In the example on the left, a simple oneto-one correspondence mapping is used, mapping the first two (out of three) segments of the demonstrator's arms and legs to the two segments of the four imitator's legs. In the second example on the right, the demonstrator's first segment of the left arm is mapped on the imitator's tail, and the demonstrator's first two right arm segments to the neck and head of the imitator's head. This results in the demonstrator controlling 'puppeteerlike' the head and tail of the robot. The grey traces visualize the body part trajectories.

2) Multiple Mappings between Dissimilar Bodies: The model of an AIBO robot is used as an imitator in the examples shown in Fig. 3. In the first example, the right arm of the demonstrator is mapped on the right front leg of the robot, the left arm to the left front leg, the right leg to the back right leg and the left leg to the back left leg. As each of the arms and legs of the demonstrator consists of three segments, and the imitator's legs consist of two segments, only the first two segments are mapped. In the second example, the imitator's head and tail are controlled 'puppeteer-like', by mapping the first two segments of the right arm and the first segment of the left arm of the demonstrator to them, respectively. The later can be also thought as an example of using a body part of the demonstrator (the left arm) to refer to a body part of the imitator (the tail) that does not have a direct equivalent on the demonstrator's (human) body. Also, although here both bodies have "heads", it might be the case that it is more

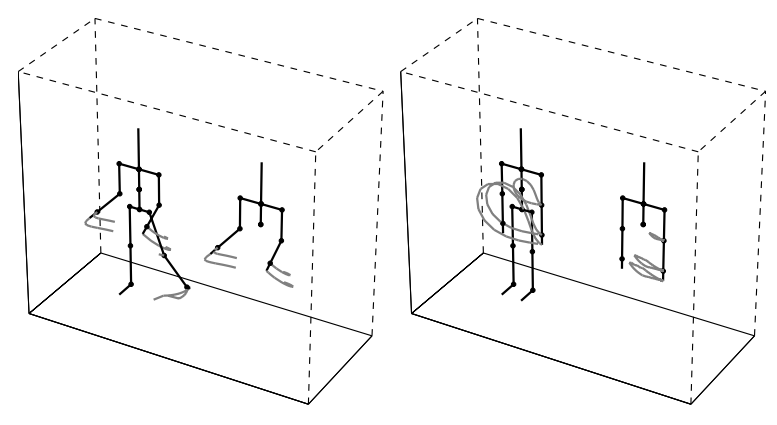

Fig. 4. Two examples of imitation across dissimilar embodiments (whole and upper torso only humanoids). The demonstrator (left in both examples) is embodied as a humanoid, while the imitator (right in both examples) is embodied as an upper torso humanoid robot.In the first example on the left, the arms of the demonstrator are mapped using a weight of one to the arms of the imitator. Note that the movement of the demonstrator's left leg is ignored as these demonstrator's DOFs are omitted (via a zero row in the correspondence matrix). In the example on the right, the same mapping is used, but the rate of movement of the imitator is severely limited, resulting in impersistence [24]. The grey traces visualize the body part trajectories.

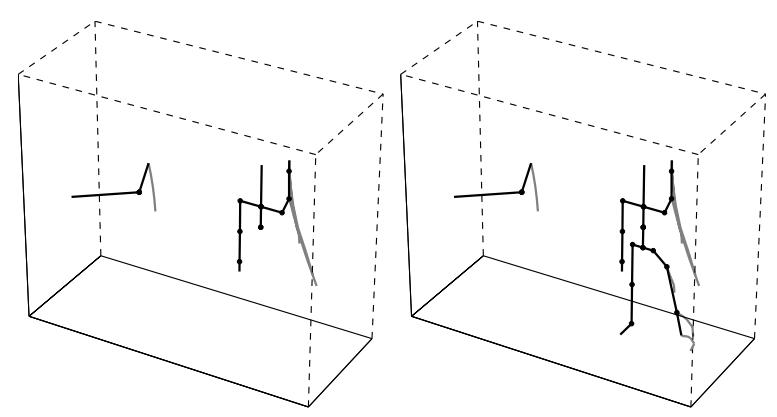

Fig. 5. Two examples of imitation across dissimilar embodiments using one-to-many correspondence mappings. The demonstrator (left in both examples) is embodied as an abstract 'letter V' shape, visualizing the three motion sensors attached to a human (one to the waist and one on each hand), while the imitator (right in both examples) is embodied as a humanoid. In the example to the left, the left segment of the demonstrator is mapped with different weight values to the imitator's left arm segments. In the second example to the right, this mapping is extended by also mapping the left segment of the demonstrator with different weight values to the imitator's left leg segments. The grey traces visualize the body part trajectories.

'expressive' (i.e. the motions/posture easier to be perceived by the imitator and/or performed by the demonstrator) to use the right arm to indicate the head movements.

3) Partial Mappings: An example of partial mapping is shown in Fig. 4 (left). As the imitator is an upper torso humanoid, the DOFs in the lower body parts of the (whole humanoid) demonstrator are ignored (via zero rows in the matrix), with a unity one-to-one mapping used for the upper body.

4) One-to-Many Mappings: When the perception of the demonstrator by the imitator is limited, a complex one-tomany correspondence mapping could be used. Assuming a human acts as a demonstrator, but the system and the imitator are provided with the coordinates of only three motion sensors, one attached to her/his waist and one in each hand. Filtering perception through this sensory apparatus yields a reduced representation of the demonstrator embodiment that 

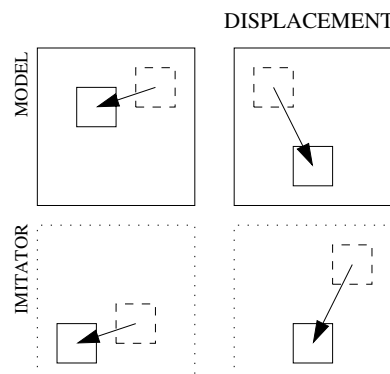

Relative Displacemen

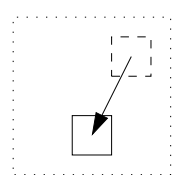

Absolute Position
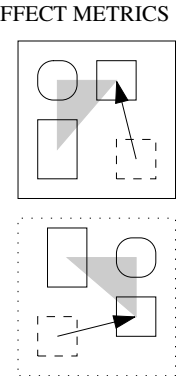

Relative Position
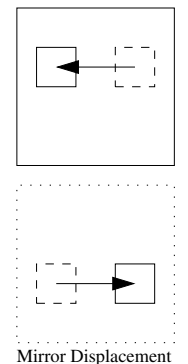

Fig. 6. A selection of displacement effect metrics. To measure the discrepancy between object displacements, the relative displacement, absolute position, relative position or mirror displacement effect metrics can be used. The first row shows four examples of effects demonstrated by the model. The second row shows the way the corresponding object (in a different workspace) needs to be moved (from dashed to solid outline) by an imitator to match the corresponding effects according to each metric. The grey triangles are superimposed to show that for the relative position effect metric, the relative final positions of the objects are the same.
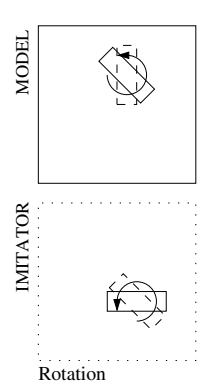

ANGULAR EFFECT METRICS
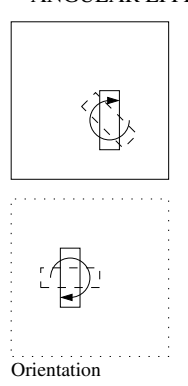

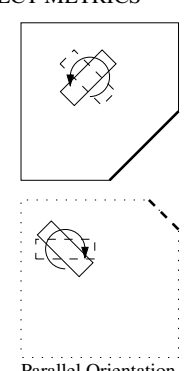

Parallel Orientation
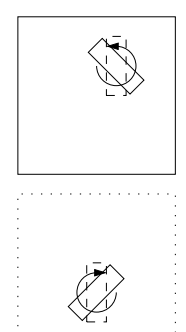

Mirror Rotation

Fig. 7. A selection of angular effect metrics. To measure the discrepancy between object displacements, the rotation, orientation, parallel orientation or mirror rotation effect metrics can be used. The first row shows four examples of effects demonstrated by the model. The second row shows the way the corresponding object (in a different workspace) needs to be rotated (from dashed to solid outline) by an imitator to match the corresponding effects according to each metric. Note that the shape of the two workspaces in the parallel orientation example is different, and the objects align with the highlighted diagonal edge.

can be modelled as a ' $\mathrm{V}$ ' letter shape kinematic model'. The $S_{j}$ and $A_{j}$ of each arm segment of this ' $\mathrm{V}$ ' embodiment could be mapped on each of the segments of the corresponding arms of a humanoid imitator, using different weights. As a result, as the human demonstrator (not shown in Fig. 5) lifts her/his left arm, the left segment of the ' $\mathrm{V}$ ' model also rises and the left arm of the humanoid imitator rises as well.

The two cases shown in Fig. 5 are presented here only as indicative examples of complex one-to-many mappings and were hand-coded.

\section{EFFECT METRICS}

Effects can be defined as changes to the body-world

\footnotetext{
${ }^{5}$ Note that as the human moves her/his arms around, the lengths of the two segments of the ' $\mathrm{V}$ ' will change accordingly and not remain constant. But this can be ignored since, for the correspondence mapping, the important parameters are the angles. These can be found from the (relative to the waist sensor) Cartesian coordinates of each arm sensor.
}

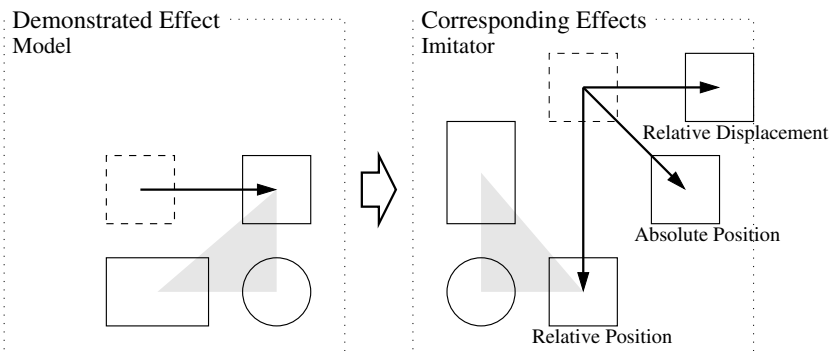

Fig. 8. Depending on the effect metric used, qualitatively dissimilar imitative behaviours can result from dissimilar object configurations (here position only). The figure illustrates three examples of using different displacement effect metrics.

relationship (e.g. location) of the agent and/or to positions, orientations and states of external objects.

Towards a characterization of the space of effect metrics, i.e. those that relate to the manipulation of objects (rather than, say, body postures or limb movements), we have explored absolute/relative angle and displacement aspects and focused on overall arrangement and the trajectory of manipulated objects [16]. Focusing on aspects of orientation and displacement of the manipulated objects, two types of effect metrics can be used, displacement and angular. The first type relates an object's movement and position in the workspace (see Figure 6), and the second type to the object's orientation (see Figure 7). Using these metrics, one can evaluate the similarity between the effects on the environment (object displacement and/or rotation) of the model and the imitator, without considering the state or the actions of the agents that caused them (cf. [7]).

Some examples of circumstances where each of these metrics can be useful (in the context of setting up a dining table) would be placing a salad bowl or the main plate in the center of the table (absolute position), arranging the forks and knives next to the plates (relative position and orientation) or (having placed a set of plates, silverware and glasses at each seat) repeating the dining arrangement for each person (relative displacement and rotation).

If the objects start from the same positions in the imitator's workspace as in the demonstrator's workspace, all the displacement effect metrics become equivalent (i.e. using any of them, the same trajectories will be generated); similarly if the objects start in the same orientations all the angular effect metrics become equivalent (the objects rotate in the same way as in the demonstration). But if the objects start in a dissimilar initial configuration (positions and/or orientations) to that of the demonstration, the choice of metrics affects qualitatively the character of the resulting imitative behaviour (see Figure 8). See [16] for precise mathematical definitions of these effect metrics, as well as for the capacity to use them in generalizing imitative behaviours across different initial configurations and applications.

\section{DISCUSSION}

A robot capable of imitating a demonstrated behaviour by another agent (artificial or human) should be able to choose 
to match one (or a weighted combination) of different aspects of that behaviour, namely the actions (motions), states (postures) and effects (on the environment). To evaluate the similarity of the demonstration with the imitation attempts, appropriate metrics, such as the ones presented in sections IV and $\mathrm{V}$ above, should be used.

We have shown how partial, mirror symmetric, one-toone, one-to-many, many-to-one and many-to-many body mappings can be characterized by (linear) correspondence matrices. These correspondences induce an infinite variety of absolute and relative state and action metrics that can be used to guide robotic imitation across dissimilar embodiments - even radically different ones in which neither the size of body parts, nor their type, nor number of DOFs need be preserved. The study of non-linear correspondences for achieving matching behaviour in states, actions and/or effects would extend this set of metrics. Currently the correspondence mapping is given, but finding the correspondence can be approached using reinforcement learning and an experiential history (adding memory), as in our previous work with the ALICE generic imitation framework [8], [9]. Future work would naturally also address the derivation of, and switching between, appropriate correspondence mappings depending on the needs of the imitator agent in the social and task context. A developed system could eventually serve as a correspondence engine for imitation learning, incorporating aspects of discovering what to imitate, depending on context and interaction history.

The relative/absolute position and rotation of objects are important aspects of a demonstrated task to match (or not) according to effect metrics, depending on the state of the objects in the environment and the context. The exploratory characterization of the space of effect metrics reveals that matching of results is a more sophisticated issue that generally acknowledged. This wide range of possible effect metrics illustrates that even the effect aspect of the correspondence problem for human-robot interaction by itself is already quite complex. Goal extraction in terms of effect metrics and granularity may have many different solutions that might not all be appropriate according to the desired results or context. This creates particular problems and challenges for sub-goal and metric extraction systems that can be used in programming robots by demonstration. The use of repeated demonstrations [10], saliency detection [11] and goal-marking via deixis and non-verbal signaling by humans [12], [13], [14] may help contribute solutions to these problems.

\section{REFERENCES}

[1] J. Demiris and G. Hayes, "Imitation as a dual-route process featuring predictive and learning components: A biologically-plausible computational model," in Imitation in Animals and Artifacts, K. Dautenhahn and C. L. Nehaniv, Eds. MIT Press, 2002, pp. 327-361.

[2] C. Breazeal and B. Scassellati, "Robots that imitate humans," Trends in Cognitive Science, vol. 6, pp. 481-487, 2002.

[3] A. Billard, "Learning motor skills by imitation: a biologically inspired robotic model," Cybernetics and Systems, vol. 32, no. 1-2, pp. 155193, 2001.
[4] M. N. Nicolescu and M. M. Matarić, "Learning and interacting in human-robot domains," IEEE Transactions on Systems, Man, and Cybernetics, Part A, vol. 31, no. 5, pp. 419-430, 2001.

[5] S. Schaal, "Is imitation learning the route to humanoid robots?" Trends in Cognitive Sciences, vol. 3, no. 6, pp. 233-242, 1999.

[6] Y. Kuniyoshi, M. Inaba, and H. Inoue, "Learning by watching: Extracting reusable task knowledge from visual observations of human performance," IEEE Trans. Robot. Automat., vol. 10, pp. 799-822, November 1994.

[7] C. L. Nehaniv and K. Dautenhahn, "Mapping between dissimilar bodies: Affordances and the algebraic foundations of imitation," in Proceedings European Workshop on Learning Robots 1998 (EWLR7), Edinburgh, 20 July 1998, J. Demiris and A. Birk, Eds., 1998, pp. 64-72.

[8] A. Alissandrakis, C. L. Nehaniv, and K. Dautenhahn, "Towards robot cultures? - Learning to imitate in a robotic arm test-bed with dissimilar embodied agents," Interaction Studies: Social Behaviour and Communication in Biological and Artificial Systems, vol. 5, no. 1, pp. 3-44, 2004.

[9] - "Imitation with ALICE: Learning to imitate corresponding actions across dissimilar embodiments," IEEE Trans. Systems, Man \& Cybernetics: Part A, vol. 32, no. 4, pp. 482-496, 2002.

[10] A. Billard, Y. Epars, S. Calinon, G. Cheng, and S. Schaal, "Discovering optimal imitation strategies," Robotics and Autonomous Systems, vol. 47:2-3, 2004.

[11] B. Scassellati, "Imitation and mechanisms of joint attention: A developmental structure for building social skills," in Computation for Metaphors, Analogy and Agents, C. L. Nehaniv, Ed. Springer Lecture Notes in Artificial Intelligence, Volume 1562, 1999, pp. 176-195.

[12] G. Butterworth, "Pointing is the royal road to language for babies," in Pointing: Where Language, Culture, and Cognition Meet, S. Kita, Ed. Lawrence Erlbaum Assoc Inc, 2003, pp. 9-26.

[13] J. Call and M. Carpenter, "Three sources of information in social learning," in Imitation in Animals and Artifacts, K. Dautenhahn and C. L. Nehaniv, Eds. MIT Press, 2002.

[14] H. Bekkering and W. Prinz, "Goal representations in imitative actions," in Imitation in Animals and Artifacts, K. Dautenhahn and C. L. Nehaniv, Eds. MIT Press, 2002, pp. 555-572.

[15] C. L. Nehaniv, "Nine billion correspondence problems and some methods for solving them," in Proc. Second International Symposium on Imitation in Animals and Artifacts - Aberystwyth, Wales, 7-11 April 2003. Society for the Study of Artificial Intelligence and Simulation of Behaviour, 2003, pp. 93-95.

[16] A. Alissandrakis, C. L. Nehaniv, K. Dautenhahn, and J. Saunders, "An approach for programming robots by demonstration to manipulate objects: Considerations on metrics to achieve corresponding effects," in Proc. 6th IEEE International Symposium on Computational Intelligence in Robotics and Automation (CIRA '05), 2005, pp. 61-66.

[17] C. L. Nehaniv and K. Dautenhahn, "Of hummingbirds and helicopters: An algebraic framework for interdisciplinary studies of imitation and its applications," in Interdisciplinary Approaches to Robot Learning, J. Demiris and A. Birk, Eds. World Scientific Series in Robotics and Intelligent Systems, 2000, pp. 136-161.

[18] _- "Like me? - measures of correspondence and imitation," Cybernetics and Systems, vol. 32, no. 1-2, pp. 11-51, 2001.

[19] - "The correspondence problem," in Imitation in Animals and Artifacts, K. Dautenhahn and C. L. Nehaniv, Eds. MIT Press, 2002, pp. 41-61.

[20] K. Dautenhahn and C. L. Nehaniv, "An agent-based perspective on imitation," in Imitation in Animals and Artifacts, K. Dautenhahn and C. L. Nehaniv, Eds. MIT Press, 2002, pp. 1-40.

[21] S. Calinon and A. Billard, "Stochastic gesture production and recognition model for a humanoid robot," in IEEE/RSJ Intl Conference on Intelligent Robots and Systems (IROS), 2004.

[22] L. M. Herman, "Vocal, social, and self imitation by bottlenosed dolphins," in Imitation in Animals and Artifacts, K. Dautenhahn and C. L. Nehaniv, Eds. MIT Press, 2002, pp. 63-108.

[23] J. Nadel, C. Guerini, A. Peze, and C. Rivet, "The evolving nature of imitation as a format of communication," in Imitation in Infancy, J. Nadel and G. Butterworth, Eds. Cambridge, 1999, pp. 209-234.

[24] J. Saunders, C. L. Nehaniv, and K. Dautenhahn, "An experimental comparison of imitation paradigms used in social robotics," in Proc. IEEE RO-MAN 2004, 13th IEEE International Workshop on Robot and Human Interactive Communication, September 20-22, 2004 Kurashiki, Okayama Japan. IEEE, 2004, pp. 691-696. 